\title{
Cholesterol, Statins, and Brain Function: A Hypothesis from a Molecular Perspective
}

\section{Yeon-Kyun Shin ${ }^{1}$}

${ }^{1}$ Integrative Bioscience and Biotechnology, POSTECH, Pohang, Korea 790-784, and Department of Biochemistry, Biophysics, and Molecular Biology, lowa State University, Ames, IA 50014.

Subject areas: Phamaceutical Science

Author contribution: Yeon-Kyun Shin wrote the paper.

*Correspondence and requests for materials should be addressed to

Y.K.S. (colishin@postech.ac.kr)

Editor: Hong Gil Nam, Postech, Republic of Korea

Received March 24, 2009; Accepted March 27, 2009;

Published March 30, 2009

Citation: Shin, Y. Cholesterol, Statins, and Brain Function: A Hypothesis from a Molecular Perspective. IBC 2009,

$1(1): 2,1-3$

doi:10.4051/ibc.2009.1.0002:

Funding: Funding for the article is provided by World Class University program in Korea; US National Institute of Health grant GM-51290.

Competing interest: All authors declare no financial or personal conflict that could inappropriately bias their experiments or writing.

Copyright: This article is licensed under a Creative Commons Attribution License, which freely allows to download, reuse, reprint, modify, distribute, and/or copy articles as long as a proper citation is given to the original authors and sources.

\section{SYNOPSIS}

There is evidence that cholesterol in the brain plays an important role in the neurotransmitter release. A decrease of the cholesterol level severely hampers the activity of the membrane fusion machinery, thereby inhibiting the release. Meanwhile, the results from several clinical studies suggest that a low cholesterol level is linked to the dysfunction of some brain activities. Because the neurotransmitter release underlies the basic brain function, the combined results lead to a testable hypothesis that the cholesterol-lowering drugs may inhibit the neurotransmitter release at the synapse. Such inhibition of the release could result in impaired brain function for a limited group of people. A molecular basis for the hypothesis is discussed.

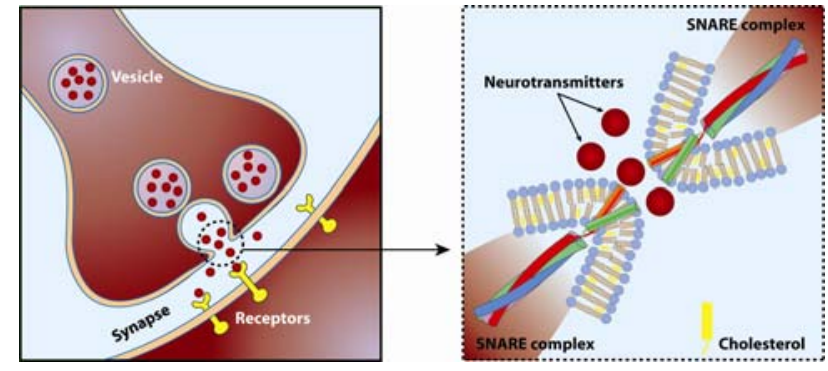

Keywords: Neurotransmitter release, SNARE, membrane fusion, synapse, raft 
Ten years ago, in 1999, BBC News reported three alarming and provocative stories on the impact of a low cholesterol level on brain function. The first story featured a large-scale 8 year-long Finnish study surveying almost 30,000 people, which revealed a strong tie between low blood cholesterol and depression and suicide (Partonen et al., 1999). The next two stories covered relatively smaller scale studies, one from Duke University and the other from Dublin, connecting the low cholesterol level to depression and dangerous behaviors, respectively (Suarez, 1999; http://news.bbc.co.uk/2/hi/health/381699.stm/).

The neurotransmitter release at the synapses underlies fundamental brain activities such as cognition, emotion, and memory. It is therefore natural to speculate that the neurotransmitter release depends on the cholesterol level in the brain. A low cholesterol level may alter the pattern of the release, which in turn perturbs the brain activities to the extent that it could lead to behavioral dysfunction, depression, suicide, and memory loss.

In a cellular level, the neurotransmitter release requires the fusion of synaptic vesicles to the presynaptic plasma membrane (Figure 1, left panel). Membrane fusion opens a passage for the neurotransmitters to be secreted to the synaptic cleft. Membrane fusion is an energy consuming process which accompanies extensive remodeling of two apposing membranes. A specialized protein machine must provide the energy required for membrane fusion. In a molecular level, the membrane fusion machinery at the synapse is a complex entity composed of many mutually interacting proteins. Among those, it is widely believed that the SNARE complex is the central component of the fusion machinery (Figure 1, right panel) (Weber et al., 1998). The release must happen in a submilisecond time scale in response to the electric signal, called the action potential. To accomplish such fast release, membrane fusion at the synapse is exquisitely controlled by the orchestrated inputs from the regulatory factors to the SNARE complex (Jahn and Scheller, 2006).

The supposition that the neurotransmitter release depend critically on cholesterol could be tested by lowering the cholesterol content in the neuronal membranes. This can be achieved by treating the neuron with $\beta$-cyclodextrin (Lang et al., 2001). This compound can extract cholesterol from cell membrane effectively, although it is incapable of extracting a desired amount of cholesterol from a selected membrane. Nevertheless, these in vivo studies support the idea that cholesterol plays an important role for the release. Alternatively, the supposition was tested in a better defined in vitro setting using a reconstituted fusion system (Tong et al., 2009). This in vitro study showed that deprivation of cholesterol from the membrane reduced the fusion activity of the SNARE fusion machinery by as much as five times. Putting together, the results from both in vivo and in vitro studies unambiguously demonstrated that cholesterol depletion causes severe inhibition of synaptic membrane fusion, thereby decreasing the neurotransmitter release.

In a molecular level, cholesterol can affect synaptic membrane fusion in a number of different ways. Firstly, cholesterol is proposed to form laterally separated membrane domains called 'rafts', in cooperation with another brain lipid sphingolipid. The rafts could play a role in driving the clustering of SNAREs into a specific membrane site (Salaun et al., 2005), which may be necessary for productive fusion. However, the evidence for the rafts in native cell membranes has not been found yet, despite they are frequently detected in model membranes. An alternative scenario is the size of the rafts might be too small to be detected with modern biophysical tools. Secondly, it is shown that cholesterol induces the clustering of the SNARE proteins by promoting self-assembly of the proteins without the rafts (Lang et al., 2001). The clustering of SNAREs is thought to be helpful for docking and fusion of vesicles. Thirdly, due to its unique inverse wedge-like molecular shape, cholesterol is thought to play a role in membrane fusion which involved curving and bending of the bilayers. For example, it is shown that cholesterol acts as a stimulator for the expansion of the fusion pore by destabilizing highly curved small pore (Biswas et al., 2008). Finally, a recent EPR study revealed that cholesterol promotes the conformational change of the transmembrane domain of a SNARE to be favorable for membrane fusion. It is also shown that the direct modulation of the SNARE structure by cholesterol is a dominant contributor to the boosted activity of the SNARE fusion machine.

The brain is highly enriched in cholesterol: As much as one quarter of total bodily cholesterol is located in the brain despite the brain-to-body mass ratio is only a few percent. In the neuron, cholesterol makes up nearly 40 percent of total lipids of the cellular membranes (Takamori et al., 2006). How does brain maintain such a high cholesterol level against the much lower blood cholesterol level? Cholesterol carried by low density lipoprotein (LDL) in the circulation cannot pass through the blood-brain barrier.
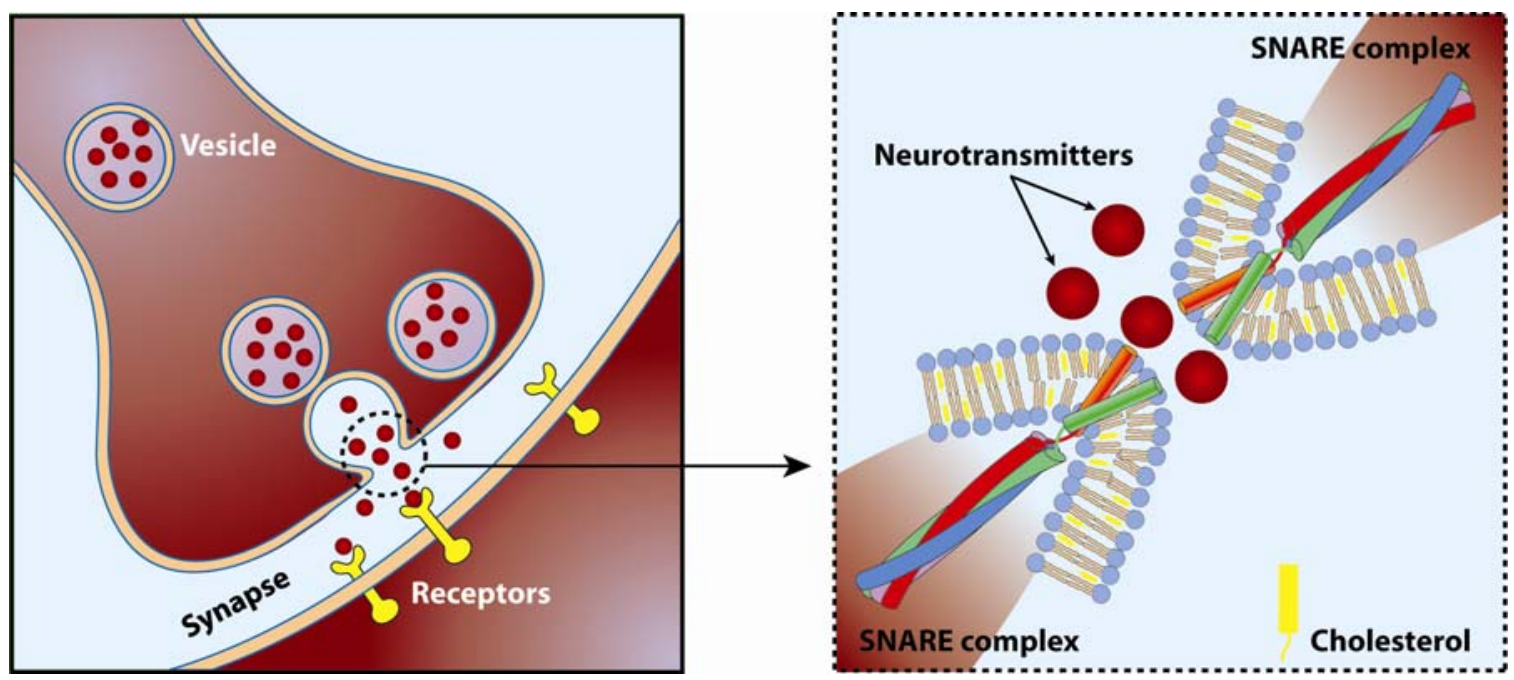

Figure 1. A schematic model for the neurotransmitter release at the synapse. Left panel, Synaptic vesicles fuse with the presynaptic plasma membrane in response to the action potential. Right panel, the core fusion machinery, the SNARE complex, drives membrane fusion, which opens the passage of the neurotransmitters from the vesicle to the synaptic cleft. Cholesterol plays a role in keeping the SNARE structure in the membrane to be favorable for membrane fusion. 
This blockage may work favorably for the non-equilibrium enrichment of cholesterol in the brain. Brain cholesterol is synthesized locally in glial cells and fed directly to the neuronal cell membranes (Vance, et al., 2005). As blood cholesterol does in the liver, brain cholesterol also turns over and it is excreted from the brain in several forms of hydroxylcholesterol (Bjorkhem et al., 2001). The delicate balance between synthesis and excretion is necessary to maintain a steady-state high cholesterol level in the brain.

How tolerable is the neurotransmitter release at the synapse to the reduced cholesterol content in neuronal cell membranes? The data that might help answer this question is almost non-existent. However, a recent in vitro study gives some idea on this important question: full 40 mole percent of cholesterol is required to maintain the high level activity of the membrane fusion machinery (Chang et al., 2009). The activity drops very quickly when cholesterol is decreased below the high natural level. This limited data suggests that the high cholesterol content in the neuronal membrane is crucial for the proper release of the neurotransmitter at the synapse.

Paradoxically, however, high blood cholesterol in the body is believed to be the primary cause of atherosclerosis and heart diseases. Public awareness of the benefit of lowering blood cholesterol has driven the widespread use of statin drugs. Statins cut cholesterol synthesis by blocking hydroxy-methylglutarylcoenzyme A reductase. Statins can go across the blood-brain barrier. Thus, we hypothesize that statins not only block the synthesis of bodily cholesterol in the liver, but they also block cholesterol synthesis in the brain in the same way. We believe that the statin use could decrease the cholesterol level in the neuronal membranes below the level required for the full activity of the membrane fusion machinery, whereby reducing the neurotransmitter release. Inhibition of the release could influence the brain functions such as cognition, emotion, decision making, and memory for a limited group of people. A recent study from University of California, San Diego supports our hypothesis (http://www.statineffcts.com/info/). This new study documented the link between statins and the reversible cognitive and memory problems.

For healthy brains of most people, inhibition of cholesterol synthesis by the statin use could be balanced by the reduction of cholesterol turnover by a yet unknown mechanism. Such counterbalance could help maintain the necessary steady-state cholesterol level in the brain. Further investigations are certainly necessary to understand such mechanisms related to cholesterol. On other note, the most common side effect of statins is the muscle pain. We speculate that this might be due partly to the impaired fusion machinery under the reduced cholesterol level at the nerve terminal.

Ten years after the three alarming BBC reports on the danger of the low cholesterol level it is now almost ubiquitously believed that benefits of lowering the blood cholesterol level outweighs the side effects of statins significantly. However, recent biochemical and clinical results suggest that the prevailing public notion must be reassessed even if the present hypothesis may apply only to the limited cases

\section{Acknowledgments}

I would like to thank Mina Shin for the figure and Dr. Mal-Gi Choi for editorial help. This work was supported by the grant from World Class University and by the US National Institute of Health grants GM-51290.

\section{References}

Biswas, S., Yin, S. R., Blank, P. S., and Zimmerberg, J. (2008) Cholesterol promotes hemifusion and pore widening in membrane fusion induced by influenza hemagglutinin. J. Gen. Physiol. 131, 503-513.

Bjorkhem, I., Andersson, U., Ellis, E., Alvelius, G., Ellegard, L., Diczfalusy, U., Sjovall, J., and Einarsson, C. (2001). From brain to bile. Evidence that conjugation and omega-hydroxylation are important for elimination of 24S-hydroxycholesterol (cerebrosterol) in humans. J. Biol. Chem. 276, 37004-37010.

Chang, J., Kim, S. A., Lu, X., Su, Z., Kim, S. K., and Shin, Y. K. (2009). Fusion step-specific influence of cholesterol on SNAREmediated membrane fusion. Biophys. J. 96, 1839-1846.

http://news.bbc.co.uk/2/hi/health/381699.stm.

http://www.statineffects.com/info/.

Jahn, R., and Scheller, R. H. (2006). SNAREs--engines for membrane fusion. Nat. Rev. Mol. Cell Biol. 7, 631-643.

Lang, T., Bruns, D., Wenzel, D., Riedel, D., Holroyd, P., Thiele, C., and Jahn, R. (2001). SNAREs are concentrated in cholesteroldependent clusters that define docking and fusion sites for exocytosis. EMBO J. 20, 2202-2213.

Partonen, T., Haukka, J., Virtamo, J., Taylor, P. R., and Lonnqvist, J. (1999). Association of low serum total cholesterol with major depression and suicide. Brit. J. Psychiat. 175, 259-262.

Salaun, C., Gould, G. W., and Chamberlain, L. H. (2005). Lipid raft association of SNARE proteins regulates exocytosis in PC12 cells. J. Biol. Chem. 280, 19449-19453.

Suarez, E. C. (1999). Relations of trait depression and anxiety to low lipid and lipoprotein concentrations in healthy young adult women. Psychosom. Med. 61, 273-279.

Takamori, S., Holt, M., Stenius, K., Lemke, E. A., Gronborg, M., Riedel, D., Urlaub, H., Schenck, S., Brugger, B., Ringler, P., Muller, S. A., Rammner, B., Grater, F., Hub, J. S., De Groot, B. L., Mieskes, G., Moriyama, Y., Klingauf, J., Grubmuller, H., Heuser, J., Wieland, F., and Jahn, R. (2006). Molecular anatomy of a trafficking organelle. Cell 127, 831-846.

Tong, J., Borbat, P. P., Freed, J. H., and Shin, Y. K. (2009). A scissors mechanism for stimulation of SNARE-mediated lipid mixing by cholesterol. Proc. Natl. Acad. Sci. USA.

Vance, J. E., Hayashi, H., and Karten, B. (2005). Cholesterol homeostasis in neurons and glial cells, Semin. Cell Dev. Biol. 16, 193-212.

Weber, T., Zemelman, B. V., McNew, J. A., Westermann, B., Gmachl, M., Parlati, F., Sollner, T. H., and Rothman, J. E. (1998). SNAREpins: minimal machinery for membrane fusion. Cell 92, 759-772. 